\title{
Ensaio clínico, aberto, controlado sobre a adição de brometo de ipratrópio ao fenoterol no tratamento da crise de asma em adultos*
}

\author{
Solange Diccini ${ }^{1}$, Clystenes Odyr Soares Silva ${ }^{2}$, Jorge Nakatanl², Carlos Alberto de Castro Pereira ${ }^{3}$
}

No tratamento da crise de asma, empregam-se doses repetidas de drogas $\beta_{2}$-agonistas por via inalatória. 0 efeito da adição do brometo de ipratrópio (BI) ao $\beta_{2}$-agonista é controverso em adultos. O bjetivo: Avaliar se adição de $\mathrm{BI}$ ao fenoterol, em tratamentos inalatórios repetidos, induz a maior broncodilatação, com reversibilidade da crise e alta da emergência em pacientes em crise grave de asma. Local do estudo: Serviço de Pronto-A tendimento de Pneumologia, Disciplina de Pneumologia da Unifesp-Hospital São Paulo, no período de julho de 1995 a fevereiro de 1997 . Tipo de

estudo: Aberto, randomizado, paralelo. Alta da emergência determinada pelo $V_{E F}$ e PFE $\geq 60 \%$ do previsto.

Casuística e métodos: Cento e vinte pacientes em crise de asma foram divididos em dois grupos $(\mathrm{N}=60)$ : fenoterol (F) e brometo de ipratrópio + fenoterol (BIF) com VEF 1 e PFE $\leq 50 \%$ do previsto. Cada grupo recebeu três tratamentos inalatórios, através de nebulímetro e câmara de expansão, administrados em intervalos de 30 minutos. No grupo F foram administrados 4 jatos de fenoterol $(400 \mathrm{mcg})$ e no grupo BIF, $160 \mathrm{mcg}$ de BI e $400 \mathrm{mcg}$ de fenoterol (4 jatos).

Resultados: A média ( \pm DP) do PFE basal $(F=36 \pm 7 \%$ vs. BIF $=35 \pm 9 \%$ previsto $)$ e do VEF basal $^{(F}=33 \pm 9 \%$ vs.

$\mathrm{BIF}=32 \pm 9 \%$ ). Trinta e dois pacientes no grupo $\mathrm{F}$ e 33 pacientes no grupo BIF tiveram alta após tratamentos inalatórios. $O \mathrm{VEF}_{1}$ e PFE ao final dos tratamentos inalatórios foram, respectivamente, $\mathrm{F}=60 \pm 13 \%$ vS. BIF $=61 \pm$ $11 \%$ e $F=74 \pm 18 \%$ vs. BIF $=77 \pm 13 \%$ (NS). Conclusão: A adição de brometo de ipratrópio ao fenoterol resulta em efeito funcional insignificante e sem impacto clínico no tratamento da crise de asma em adultos.

(J Pneumol 1999;25(6):301-308)

Open, controlled clinical assay of the addition of ipratropium bromide to fenoterol in the treatment of acute asthma crisis in adults

Repeated dosis of inhaled $\beta_{2}$-agonists have been used in the treatment of acute asthma. The effect of added ipratropium bromide (IB) to $\beta_{2}$-agonist is controversial in adults. Objective: To evaluate if addition of IB to fenoterol, in repeated doses, induces a greater bronchodilation, a greater reversion of the attack, and discharge from emergency unit in adults with acute severe asthma. Setting: Pneumology Emergency Department, Unifesp-Hospital São Paulo, in the period from J uly 1995 to February 1997. Type of study: Open, randomized and parallel study. Discharge from the hospital: $\mathrm{FEV}_{1}$ and PEF $\geq 60 \%$ of the predicted value. Methods: 120 patients with $\mathrm{FEV}$ and PEF $\leq 50 \%$ of the predicted value were divided into two groups $(\mathrm{N}=60)$ : fenoterol $(\mathrm{F})$ and ipratropium bromide + fenoterol (IBF).

Each group received inhalation treatment through a metered-dose inhaler (MDI) attached to a holding chamber, administered at 30-minute interval, for a total of three treatments. In the group $\mathrm{F}$ four puffs of fenoterol $(400 \mu \mathrm{g})$ were administered, and in the IBF group, $160 \mu \mathrm{g}$ of BI and $400 \mu \mathrm{g}$ of fenoterol (four puffs). Results: The patients did not differ from basal PEF ( $F=36 \pm 7 \%$ vs IBF $=35 \pm 9 \%$ predicted) and basal $F E V_{1}$ ( $F=33 \pm 9 \%$ vs IBF $=32 \pm$ $9 \%$ predicted). Thirty-two patients of group $\mathrm{F}$ and 33 of group IBF were discharged from hospital after the inhalation treatment. The final FEV 1 and PEF after inhalation treatments were $F=60 \pm 13 \%$ vs IBF $=61 \pm 11 \%$ e F $=74 \pm$ $18 \%$ vs IBF $=77 \pm 13 \%$ (NS). Conclusion: The addition of ipratropium bromide to fenoterol results in insignificant functional effect and without clinical impact in the treatment of acute asthma in adults.

* Trabalho realizado no Setor de Pronto-A tendimento da Disciplina de Pneumologia da Unifesp-Hospital São Paulo. Este estudo é parte da tese de Doutorado "Ensaio clínico controlado e meta-análise sobre a adição de brometo de ipratrópio ao fenoterol no tratamento da crise grave de asma", para obtenção do título de Doutora em Ciências Pneumológicas, 1997.

1. Enfermeira; Professora A djunta do Departamento de Enfermagem da Universidade Federal de São Paulo/ EPM; Doutora em Ciências Pneumológicas, Unifesp-EPM.
2. Professor Adjunto da Disciplina de Pneumologia da Unifesp-EPM; Doutor em Medicina, Unifesp-EPM.

3. Médico Assistente da Disciplina de Pneumologia da Unifesp-EPM; Doutor em Medicina, Unifesp-EPM.

Endereço para correspondência - Solange Diccini, Av. Jacutinga, 632/84, Moema - 04515-030 - São Paulo, SP.

Recebido para publicação em 29/3/99. Reapresentado em 14/6/99. A provado, após revisão, em 18/6/99. 
Descritores - Fenoterol. N-isopropilatropina. Estado asmático. Terapia respiratória.

Key words - Fenoterol. Ipratropium. Status asthmaticus. Respiratory therapy.

\section{INTRODUÇÃO}

Estratégias de tratamento da crise de asma são dirigidas para reverter o broncoespasmo e aliviar a inflamação das vias aéreas. Diversos consensos sobre o manejo dos pacientes asmáticos abordaram 0 atendimento destes em serviços de emergência ${ }^{(1-4)}$.

As drogas $\beta_{2}$-agonistas têm eficácia imediata comprovada na crise de asma. A administração dos $\beta_{2}$-agonistas na crise deve ser feita por via inalatória, devido a seu rápido início de ação, em torno de cinco minutos, atingindo o pico máximo em 15 minutos e com duração de três a seis horas ${ }^{(3-6)}$.

$\mathrm{Na}$ emergência, a administração de rotina da terapia inalatória é realizada por nebulização de jato (NJ). A lguns estudos demonstraram que, se bem usado, o nebulímetro acoplado à câmara de expansão (NBL-CE) é tão eficiente quanto a $\mathrm{N}{ }^{(7)}$. Há correlação da dose de $\beta_{2}$-agonista fornecida por $\mathrm{N}$ J e aquela com o NBL. Por curvas dose-resposta, o efeito máximo é obtido na asma com doses de $2,5 \mathrm{mg}$ e $400 \mathrm{mcg}$, respectivamente. A dose ótima do $\beta_{2}$-agonista na crise de asma, fornecida através de NBL-CE para produção de máxima broncodilatação, situa-se entre 800 e 1.600 mcg (8 a 16 jatos) de salbutamol(8-10).

O brometo de ipratrópio (BI) é um derivado amônio quaternário da atropina. 0 início de ação do BI é mais lento do que 0 dos agentes $\beta_{2}$-agonistas, de 15 a 30 minutos $^{(11)}$. Estudos de dose-resposta realizados em pacientes com obstrução leve ao fluxo aéreo sugeriram a dose de $40-80 \mathrm{mcg}$ por NBL para resposta máxima ao $\mathrm{BI}^{(12,13)}$. Em pacientes com doença obstrutiva crônica moderada e grave foi sugerida a dose de 160 mcg por NBL ${ }^{(14)}$, porém nos com crise de asma não há estudos em relação à dose ótima.

Rebuck et al. (15) publicaram um estudo multicêntrico, em que foram avaliados pacientes com DPOC e asma, tratados na emergência, de maneira randomizada, com dose única de BI $(500 \mathrm{mcg})$, fenoterol $(1,25 \mathrm{mg})$ ou associação de ambos, por N . O $\mathrm{VEF}_{1}$ inicial era $<70 \%$ do valor previsto. A espirometria foi repetida após 45 e 90 minutos. Nos 97 portadores de asma, verificou-se que o fenoterol (F) teve efeito significativamente maior que $0 \mathrm{BI}$ isolado e que a associação foi melhor que os dois broncodilatadores dados em separado. As respostas dos pacientes com $\mathrm{VEF}_{1}$ acima e abaixo de $1,0 \mathrm{~L}$ foram comparadas; O VEF , após 90 minutos, elevou-se $0,25 \mathrm{~L}$ com a combinação em relação ao $\mathrm{F}$ isolado no grupo mais obstruído. Nos pacientes com VEF ${ }_{1}$ acima de 1,0L 0 incremento em relação ao $\mathrm{F}$ foi de apenas $0,11 \mathrm{~L}$. Os autores concluíram que a associação é especialmente indicada em pacientes mais gravemente obstruídos. Dois problemas sé-
Siglas e abreviaturas utilizadas neste trabal ho

BI - Brometo de ipratrópio

BIF - Brometo de ipratrópio + fenoterol

NJ - Nebulização de jato

NBL-CE - Nebulímetro acoplado à câmara de expansão

$\mathrm{F}$ - Fenoterol

RECM - Retração do esternoclidomastóideo

$P P$ - Pulso paradoxal

$\mathrm{SpO}_{2}$ - Saturação de oxigênio

$f$ - Freqüência respiratória

FC - Freqüência cardíaca

PAS - Pressão arterial sistólica

PAD - Pressão arterial diastólica

CPT - Capacidade pulmonar total

$V E F_{1}$ - Volume expiratório forçado no 1 ㅇ segundo

PFE - Pico de fluxo expiratório

rios a respeito deste estudo são: a diferença basal no grau de obstrução entre os grupos e a dose baixa e única de $\mathrm{F}$.

Em 1990, uma revisão não sistemática foi publicada(16). Dezoito ensaios clínicos feitos com anticolinérgicos na asma aguda foram criticamente revistos. Várias conclusões foram retiradas: os anticolinérgicos isolados produzem broncodilatação modesta na asma aguda grave, mas não tão consistente quanto os $\beta_{2}$-agonistas; os anticolinérgicos produzem broncodilatação adicional aos $\beta_{2}$-agonistas em estudos de doses únicas e múltiplas; a broncodilatação adicional obtida, de 10 a $20 \%$, parece ser maior nos pacientes mais gravemente obstruídos, não resulta em melhor desfecho para a crise e não foi avaliada contra a administração de doses altas e freqüentes de $\beta_{2}$-agonistas. A recomendação final foi a de que esses agentes devem ser de segunda linha no tratamento da crise de asma, exceto possivelmente para aqueles que se apresentam com obstrução mais grave ( $\mathrm{PFE}<35 \%$ do previsto). N esta revisão, estudos de diversas concepções (cruzados, paralelos, envolvendo doentes internados ou não) foram incluídos. O desfecho das crises não foi avaliado contra dados objetivos.

Lanes et al. ${ }^{(17)}$ analisaram em conjunto três ensaios randomizados, de concepção similar, envolvendo a administração de dose única de $2,5 \mathrm{mg}$ de salbutamol por NJ contra salbutamol adicionado de BI $(0,5 \mathrm{mg})$, em 1.064 pacientes. 0 $\mathrm{VEF}_{1}$ foi medido aos 45 e 90 minutos. A pós 45 e 90 minutos a diferença na mediana do $\mathrm{VEF}_{1}$ a favor do tratamento combinado foi de 40 e $47 \mathrm{ml}$, respectivamente. No grupo tratado com a combinação, o $\mathrm{VEF}_{1}$ aumentou de maneira similar naqueles com valores iniciais acima e abaixo de 1,0L. A necessidade de tratamento adicional e hospitalização foi avaliada retrospectivamente e decidida sem dados objetivos e não diferiu significantemente entre os grupos.

As diferenças nos estudos refletem-se nos consensos sobre asma. Em três consensos recentemente publicados, a posição do BI é comentada na crise. 0 consenso norte-americano(2) recomenda a associação de altas doses de $\beta_{2}$-ago- 
nistas e BI por nebulização a cada 20 minutos, durante uma hora, sempre que o $\mathrm{VEF}_{1}$ ou o PFE estiver abaixo de $50 \%$ do previsto. $O$ consenso britânico ${ }^{(1)}$ recomenda que 0 BI seja adicionado ao tratamento em doentes com risco de vida, com PFE $<33 \%$ do previsto e nos pacientes sem melhora após 0 uso de $\beta_{2}$-agonista. 0 consenso canadense ${ }^{(3)}$, sobre asma aguda, recomenda que BI deveria ser adicionado ao $\beta_{2}$ agonista em casos de asma grave, $\mathrm{VEF}_{1}<1,0 \mathrm{~L}$ ou PFE $<$ $140 \mathrm{~L} / \mathrm{min}$. Oito ensaios são citados, de nível I, para suportar essa conclusão. $O$ consenso observou que nenhum dos estudos administrou BI por NBL-CE.

Muitos estudos publicados utilizaram dose única de BI, porém o tratamento atual da crise envolve doses repetidas de $\beta_{2}$-agonista; portanto, a relevância desses estudos pode ser questionada. Além disso, o desfecho de maior importância na crise é a redução de tratamentos adicionais e internações. A maioria dos estudos avalia apenas o efeito funcional.

0 presente trabalho teve como objetivo avaliar se adição do brometo de ipratrópio ao fenoterol, em tratamentos inalatórios repetidos, induz maior broncodilatação, permitindo maior reversibilidade da crise e alta da emergência em pacientes em crise grave de asma.

\section{CASUÍSTICA E MÉTOdOS}

$O$ estudo foi aberto, randomizado e com grupos paralelos, tendo como desfecho principal a alta da emergência após o alcance e a estabilização de valores funcionais predetermina-

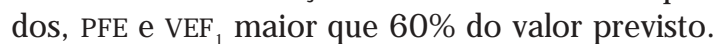

\section{Pacientes}

Foram analisados 120 pacientes adultos, de ambos os sexos, com o diagnóstico de crise de asma, assistidos no Pronto-A tendimento da Disciplina de Pneumologia da Universidade Federal de São Paulo, durante o período de julho de 1995 a fevereiro de 1997. Todos os pacientes foram informados sobre os objetivos do estudo e concordaram em participar dele. 0 trabalho foi aprovado pela Comissão de Ética do Hospital. Os pacientes preencheram os seguintes critérios de inclusão: 1) Idade de 18 a 70 anos; 2) Diagnóstico de asma feito por médico com história de, pelo menos, duas crises prévias de dispnéia e chiado, aliviadas com broncodilatador; 3) Surgimento ou agravamento dos sintomas de asma nos últimos dias ou horas; 4) PFE e VEF $F_{1}$ menor que $50 \%$ do valor previsto. Os pacientes foram excluídos se: fumantes acima de 50 anos de idade ou com mais de 30 anos-maço de tabagismo; com tosse e expectoração crônica por mais de três meses/ ano durante pelo menos dois anos consecutivos; mulheres grávidas ou em período de lactação; com doenças pulmonares associadas ou pregressas que resultaram em seqüelas pulmonares; com infecção respiratória aguda das vias aéreas inferiores caracterizada por pelo menos dois dos três achados: febre, expectoração purulenta ou infiltrado pulmonar ao radiograma torácico; portadores de doenças cardía- cas ou renais; com incapacidade de fazer prova de função pulmonar adequada, por não colaboração ou por estarem muito graves (em franca insuficiência respiratória, apresentando respiração toracoabdominal, com incapacidade de falar, presença de cianose), exigindo tratamento imediato, sem possibilidade de avaliação pelo protocolo; participação prévia no estudo.

A avaliação dos sinais e sintomas de gravidade da crise foi realizada através da: 1) A valiação clínica: estado geral, fala, retração do esternoclidomastóideo (RECM), pulso paradoxal (PP), sibilos e dispnéia; 2) Avaliação das medidas da função pulmonar: PFE e $\mathrm{VEF}_{1}$; 3) A valiação respiratória e hemodinâmica: saturação de oxigênio $\left(\mathrm{SpO}_{2}\right)$, freqüência respiratória (f) e cardíaca (FC), pressão arterial sistólica (PAS) e diastólica (PAD).

\section{Protocolo}

Para propósitos de randomização, após a realização das medidas de PFE e $\mathrm{VEF}_{1}$, os pacientes foram classificados em dois grupos: um grupo com crise grave e outro grupo com crise muito grave. Os pacientes do grupo com crise grave foram caracterizados por $\mathrm{VEF}_{1}$ entre 26 e $50 \%$ do valor previsto, e os pacientes do grupo com crise muito grave, por $\mathrm{VEF}_{1}$ menor que $25 \%$ do valor previsto.

Os pacientes do grupo com crise grave e muito grave foram divididos, de forma randomizada e paralela, em dois esquemas terapêuticos: um grupo recebeu fenoterol (F) e 0 outro grupo, brometo de ipratrópio mais fenoterol (BIF). Cada esquema poderia ser repetido até três vezes $(1 \mathrm{~T}, 2 \mathrm{~T}, 3 \mathrm{~T})$, com intervalo de 30 minutos entre os tratamentos.

Os pacientes do grupo com crise grave, do grupo $F$, receberam por NBL-CE, de $750 \mathrm{ml}$ (Fisonair), 4 jatos de $\mathrm{F}$ (Berotec $100 \mathrm{mcg}$, spray, Boehringer De Angeli), num total de $400 \mathrm{mcg}$. Os pacientes do grupo BIF receberam, por NBL-CE, 4 jatos, num total de $400 \mathrm{mcg}$ de $\mathrm{F}$ e de $160 \mathrm{mcg}$ de BI (Duovent spray, Boehringer De Angeli). Os pacientes do grupo muito grave receberam o dobro da dose no primeiro tratamento. 0 NBL-CE era ativado no final da expiração e o paciente era estimulado a realizar uma inspiração lenta e profunda até a CPT, mantendo uma pausa inspiratória sustentada durante 10 segundos, após o que se permitia a expiração. A guardavam-se 30 segundos para a realização de nova manobra, até que fosse completado o total de jatos de cada tratamento.

A avaliação da função pulmonar iniciada após 20 minutos do tratamento inalatório determinava o término ou a continuação do tratamento. 0 esquema terapêutico era interrompido em ambos os grupos, quando os pacientes atingiam um valor funcional preestabelecido do PFE e do $\mathrm{VEF}_{1} \geq 60 \%$ do valor previsto. A partir desses valores funcionais, os pacientes permaneciam por um período de 30 minutos em observação, sendo reavaliados ao término deste tempo, com a finalidade de detectar recaída funcional. Nos casos em que 0 PFE ou o $\mathrm{VEF}_{1}$ caíam abaixo de $60 \%$ do valor previsto, 0 
tratamento era reiniciado, retomando-se a seqüência do esquema terapêutico. Os pacientes com PFE e $\mathrm{VEF}_{1} \geq 60 \%$ do valor previsto recebiam alta com a terapêutica de manutenção adequada a cada caso.

Se após o 3 tratamento, o PFE ou o VEF ${ }_{1}$ persistiam abaixo de $60 \%$ do valor previsto, os pacientes recebiam 4 jatos adicionais dos broncodilatadores previamente administrados (do grupo F ou do grupo BIF) e aminofilina e metilprednisolona por via parenteral. A aminofilina foi administrada na dose de $3 \mathrm{mg} / \mathrm{kg}$ ou de $6 \mathrm{mg} / \mathrm{kg}$, dependendo de o paciente ter usado ou não esta droga, respectivamente, nas últimas 24 horas. A aminofilina foi diluída em $100 \mathrm{ml}$ de soro fisiológico a 0,9\% e infundida, por via intravenosa, no mínimo em 30 minutos. A metilprednisolona foi administrada na dose de $60 \mathrm{mg}$, independente do uso prévio ou não desta droga. A pós esse tratamento (4T), nova avaliação clínica e funcional foi realizada, a qual determinava a continuação da terapêutica, alta do pronto-atendimento ou internação hospitalar.

Achados indicativos de gravidade de asma e da crise

A intensidade da gravidade da crise de asma foi graduada a partir de um escore, obtido pela soma dos pontos, de 0 a 7 , considerando as variáveis que se correlacionam com 0 nível de obstrução ao fluxo aéreo. Cada achado recebeu uma nota: 0 - sugerindo crise leve ou moderada e 1 - sugerindo crise grave ou muito grave. O s sinais e sintomas de gravidade incluídos e sua graduação foram:

a) Fala: 0 - capaz de falar sentenças sem interrupção para inspiração; 1 - interrupção da fala em frases ou sílabas;

b) Sibilos: 0 - ausentes, localizados ou esparsos; 1 - difusos, audíveis com e sem estetoscópio, tórax silencioso;

c) Dispnéia: 0 - ausente, muito leve, leve, um pouco forte; 1 - forte, muito forte, máxima, de acordo com percepção do paciente;

d) RECM: 0 - ausente, leve; 1 - moderada, acentuada;

e) PP: 0 - ausente; 1 - presente por palpação radial;

f) FC: 0 - menor que 110 bat./ min; 1 - maior que 110 ou menor que 60 bat./ min;

g) $\mathrm{SpO}_{2}$ : 0 - maior que 93\%; 1 - menor ou igual a 93\%.

O escore foi comparado nos grupos F e BIF, na avaliação basal e após cada tratamento inalatório. Asma de alto risco foi definida pela necessidade de ventilação mecânica por crise prévia.

\section{Avaliação da função pulmonar}

A espirometria foi realizada, de acordo com as normas da ATS ${ }^{(18)}$, em espirômetro de fole acurado (Vitatrace VT 130 $\mathrm{SL})$. O v valores previstos foram os existentes para a espirometria na população brasileira(19).

Para a avaliação do PFE foi utilizado um único aparelho da marca Assess (Healthscan, Cedar Grove, NJ). No mínimo eram realizadas três medidas, sendo anotada a de maior valor, não podendo haver diferença maior do que 30L/min entre as duas melhores(20).
A monitorização da saturação de oxigênio foi feita através de oxímetro de pulso (modelo DX 2455 - Dixtal Biomédica Ind. e Com. Ltda.). Cada paciente foi monitorizado de forma contínua do início ao fim do tratamento. A $\mathrm{SpO}_{2}$ menor ou igual a 93\% implicava a administração de oxigênio, através de cateter nasal, com fluxo de $2 \mathrm{~L} / \mathrm{min}$, até o fim dos tratamentos.

\section{Reversibilidade da obstrução das vias aéreas}

A reversibilidade da obstrução ao fluxo aéreo após administração de broncodilatador foi avaliada através da variações do PFE e do VEF ${ }_{1}$ em relação aos valores previstos. Devido aos valores semelhantes de função pulmonar na avaliação inicial, outras maneiras de expressão de resposta foram calculadas, mas desconsideradas, por mostrarem resultados semelhantes ${ }^{(21,22)}$.

\section{Método estatístico}

Tomando-se por base o desvio-padrão de 0,52L do $\operatorname{VEF}_{1}$, após a inalação de drogas broncodilatadoras em estudo anterior ${ }^{(23)}$, e uma variação tida como clinicamente significativa de $0,2 L^{(24)}$, foi calculado o tamanho da amostra para $\alpha=$ 0,05 e $\beta=0,20$, sendo necessários 60 pacientes em cada grupo. Foram aplicados os seguintes testes: teste de MannWhitney para duas amostras independentes, com a finalidade de comparar os grupos F e BIF quanto aos valores do PFE e do $V_{E F}$ e respectivos incrementos em relação aos valores previstos; teste $\mathrm{t}$ de Student para duas amostras independentes, com o propósito de comparar os grupos F e BIF, em relação à freqüência cardíaca e freqüência respiratória; teste de aderência para duas amostras de Kolmogorov-Smirnov, com objetivo de comparar os grupos F e BIF, em relação à percentagem de alta após cada tratamento inalatório.

Em todos os testes fixou-se em 0,05 ou $5 \%$ o nível para a rejeição da hipótese de nulidade, assinalando-se com um asterisco os valores significantes.

\section{Resultados}

Cento e vinte pacientes foram incluídos no estudo, sendo 60 alocados para o grupo F e 60 para o grupo BIF. A Tabela 1 apresenta dados demográficos, sinais e sintomas de gravidade da crise e dados funcionais dos dois grupos estudados.

$\mathrm{Na}$ avaliação basal os grupos não diferiram significantemente nos diversos parâmetros de gravidade avaliados. Igualmente, não foram observadas diferenças significantes nesses parâmetros entre os grupos F e BIF, após os tratamentos inalatórios. Vinte pacientes (34\%) do grupo F e 15 (30\%) do grupo BIF referiram como principal fator desencadeante da crise a mudança de clima; a infecção das vias aéreas superiores foi considerada para $11(18,6 \%)$ pacientes do grupo $\mathrm{F}$ e $11(22 \%)$ do grupo BIF, como a segunda causa mais freqüente. Essas diferenças não foram significantes. 
TABELA 1

D ados demográficos, sinais e sintomas da gravidade da crise e dados funcionais dos pacientes dos grupos fenoterol (F) e brometo de ipratrópio + fenoterol (BIF)

\begin{tabular}{|c|c|c|c|}
\hline & $\mathbf{F}$ & BIF & $\mathbf{P}$ \\
\hline Número & 60 & 60 & - \\
\hline Idade (anos, $\mathrm{X} \pm \mathrm{DP}$ ) & $39 \pm 16$ & $40 \pm 13$ & NS \\
\hline Sexo $(M / F)$ & $21 / 39$ & $23 / 37$ & NS \\
\hline Gravidade da asma (leve/moderada/grave) & $32 / 19 / 9$ & $35 / 11 / 14$ & NS \\
\hline Duração da crise (M d e variação; horas) & $96(3-360)$ & $72(2-336)$ & NS \\
\hline U so de $\mathrm{Bd}$ nas últimas 4 horas (Sim/Não) & $37 / 23$ & $29 / 31$ & NS \\
\hline Asma de alto risco $(\mathrm{N})$ & 6 & 6 & NS \\
\hline Freqüência respiratória (rpm, $X \pm D P)$ & $22 \pm 4$ & $23 \pm 6$ & NS \\
\hline Freqüência cardíaca (bpm, $X \pm D P)$ & $93 \pm 17$ & $94 \pm 16$ & NS \\
\hline Dificuldade para falar frases ou sílabas $(\mathrm{N}, \%)$ & $46(77)$ & $38(63)$ & NS \\
\hline Sibilos difusos ou audíveis sem estetoscópio (N, \%) & $50(83)$ & $50(83)$ & NS \\
\hline Uso de musculatura acessória (N, \%) & $54(90)$ & $49(82)$ & NS \\
\hline Dispnéia (forte, muito forte, máxima; N, \%) & $35(66,6)$ & $47(78,3)$ & NS \\
\hline Pulso paradoxal $(\mathrm{N}, \%)$ & $49(81,7)$ & $47(78,3)$ & NS \\
\hline $\mathrm{SpO}_{2}(\%, \mathrm{X} \pm \mathrm{DP})$ & $95 \pm 2$ & $95 \pm 2$ & NS \\
\hline Escore de gravidade ( $\mathrm{Md}$ e variação) & $4(0-7)$ & $4(0-7)$ & NS \\
\hline $\mathrm{PFE}(\mathrm{L} / \mathrm{min}, \mathrm{X} \pm \mathrm{DP})$ & $166 \pm 43$ & $164 \pm 43$ & NS \\
\hline PFE (\% do previsto, $\mathrm{X} \pm \mathrm{DP})$ & $36 \pm 7$ & $35 \pm 9$ & NS \\
\hline $\operatorname{VEF}_{1}(L, X \pm D P)$ & $0,97 \pm 0,33$ & $0,93 \pm 0,28$ & NS \\
\hline $\operatorname{VEF}_{1}(\%$ do previsto, $\mathrm{X} \pm \mathrm{DP})$ & $33 \pm 9$ & $32 \pm 9$ & NS \\
\hline
\end{tabular}

$\mathrm{N}=$ número absoluto; dados analisados por teste $t$ de Student, Mann-W hitney e qui-quadrado NS = não significante

TABELA 2

Escore de gravidade dos pacientes dos grupos fenoterol $(F)$ e brometo de ipratrópio + fenoterol (BIF), basal e após os tratamentos inalatórios

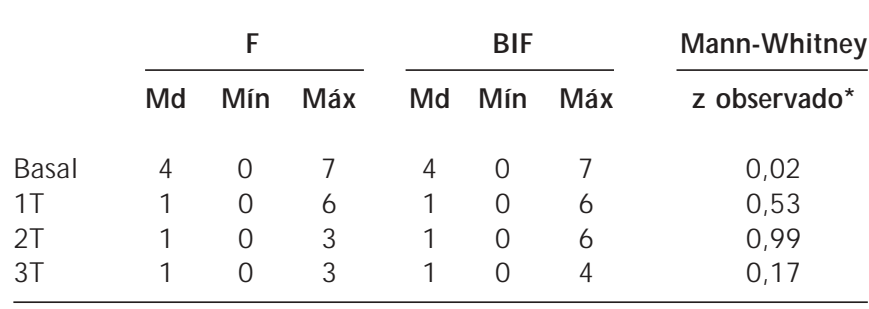

* z crítico $=1,96$

A mbos os grupos apresentaram efeitos colaterais semeIhantes e já esperados para os $\beta_{2}$-agonistas, porém foram bem tolerados pela maioria dos pacientes; tremor moderado ou acentuado foi observado em 33 pacientes do grupo $\mathrm{F}$ e 32 do grupo BIF.

Durante a avaliação basal, foi observada $\mathrm{SpO}_{2}=93 \% \mathrm{em}$ 13 pacientes $(22 \%)$ do grupo $\mathrm{F}$ e em 11 pacientes $(18 \%)$ do grupo BIF. Nesses pacientes, conforme o protocolo, foi instalado cateter nasal com fluxo de oxigênio de $2 \mathrm{~L} / \mathrm{min}$.

Antes e após os tratamentos inalatórios, o escore de gravidade não apresentou diferença estatisticamente significante entre os dois grupos (Tabela 2).

Do total de 120 pacientes, $33(27 \%)$ receberam alta após o 1 을 tratamento, $24(20 \%)$ após o 20 tratamento e $8(7 \%)$ após 030 tratamento. A o final dos três tratamentos inalató-

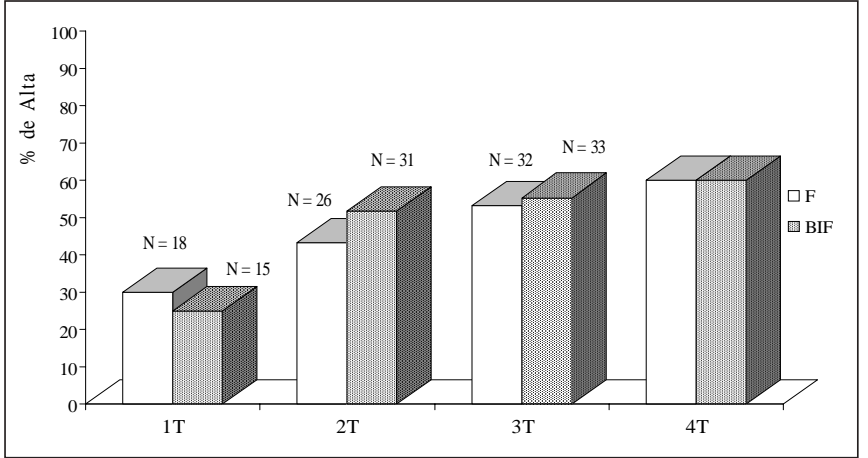

Figura 1 - Número de altas cumulativas dos pacientes do grupo fenoterol (F) e brometo de ipratrópio + fenoterol (BIF), após os tratamentos inalatórios. (KS, diferenças não significantes)

rios, $32(53 \%)$ do grupo $\mathrm{F}$ receberam alta comparados com os 33 (55\%) do grupo BIF (NS). Vinte e sete pacientes $(45,0 \%)$ do grupo F e $27(45,0 \%)$ do grupo BIF prosseguiram o tratamento com a administração de medicações parenterais. U ma paciente do grupo F não recebeu aminofilina, por ter apresentado taquicardia sinusal. Dos 27 pacientes de cada esquema terapêutico, $4(14,8 \%)$ do grupo F e $3(11,1 \%)$ do grupo BIF receberam alta após a infusão de aminofilina. 0 restante dos pacientes permaneceu no pronto-atendimento até a alta ou foram internados. Pelo teste de qui-quadrado, os pacientes dos grupos F e BIF não apresentaram diferenças estatisticamente significantes, segundo o número de altas após 01 , 2 , 3 ㅇ tratamentos inalatórios e após aminofilina (Figura 1 ). 
Dois pacientes (3\%) do esquema $\mathrm{F}$ evoluíram com recaída durante a avaliação de 30 minutos, sendo necessário retomar o protocolo. Destes pacientes, um apresentou recaída

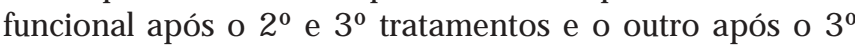
tratamento, necessitando receber aminofilina e corticóide por via parenteral. Nenhum paciente do esquema BIF apresentou recaída funcional durante a avaliação de 30 minutos.

0 teste de Mann-W hitney não mostrou diferença estatisticamente significante entre os grupos F e BIF em relação ao tempo para cada tratamento inalatório, em torno de $30 \mathrm{mi}-$ nutos, bem como no tempo total dos tratamentos inalatórios $(F, X \pm D P=68 \pm 31$ minutos; $B I F, X \pm D P=63 \pm 28$ minutos).

Os valores de PFE e $V_{E F}$ não diferiram ao final dos tratamentos inalatórios entre os dois grupos (Figura 2), e também após a infusão de aminofilina e corticosteróide IV (dados não mostrados).

Durante a avaliação basal, 9 pacientes $(15 \%)$ do grupo $\mathrm{F}$ e 10 do grupo BIF (17\%) apresentaram $\mathrm{VEF}_{1}$ menor ou igual a $25 \%$ do valor previsto e caracterizados como pacientes com crise muito grave. Em ambos os grupos, sete pacientes necessitaram receber medicação por via parenteral.

\section{DISCUSSÃO}

0 presente estudo avaliou o efeito da adição de brometo de ipratrópio ao $\beta_{2}$-agonista, utilizando nebulímetro pressurizado e tendo como desfecho principal a alta mais precoce da emergência. Não houve efeito adicional do brometo de ipratrópio.

Pacientes com valores de pico de fluxo expiratório abaixo de $50 \%$ do valor previsto e com achados clínicos de exacerbação devem ser tratados intensamente para reversão do quadro. Exacerbações de asma de tal magnitude devem ser caracterizadas como graves ${ }^{(1)}$. O VEF 1 reflete melhor 0 grau de obstrução e deve ser preferido quando disponível. Diferentes níveis de obstrução ao fluxo aéreo, de leve a muito acentuado, foram selecionados para inclusão em diferentes estudos que avaliaram o efeito da adição do Bı em crises de asma $^{(15,23)}$.

As curvas dose-resposta aos broncodilatadores são dinâmicas e dependentes do grau de obstrução presente no início do tratamento. Este fenômeno ocorre tanto para os $\beta_{2}$ agonistas como para os anticolinérgicos ${ }^{(25)}$. Devido a isso, pacientes com VEF ${ }_{1}$ abaixo de $25 \%$ foram classificados como portadores de crise muito grave e randomizados em separado no início do estudo, tendo recebido o dobro da dose inicial dos broncodilatadores.

0 estudo foi aberto. Fenoterol e a associação de fenoterol com brometo de ipratrópio são disponíveis em diferentes nebulímetros, porém de tamanhos diferentes. Nebulímetro contendo placebo não pôde ser obtido. 0 fornecimento da medicação e o registro das observações foram realizados pelo

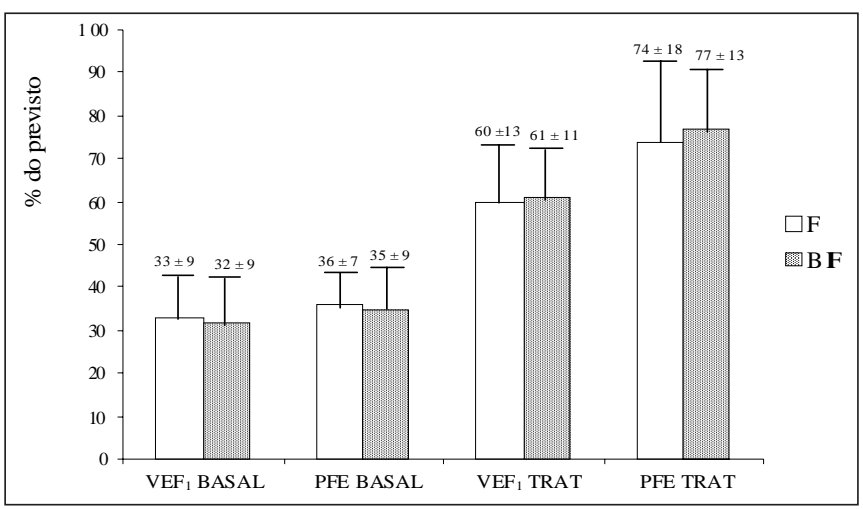

Figura 2 - Valores de VEF 1 e PFE em percentagem do previsto na avaliação basal e após os tratamentos inalatórios, associados ou não à aminofilina e metilprednisolona, expressos em média e desvio-padrão

principal investigador, treinado com especialistas habituados na avaliação de crises de asma, antes do estudo.

A alta da emergência foi avaliada pelo alcance de valores funcionais preestabelecidos. As medidas de PFE são dependentes de esforço e viés poderia ser introduzido por maior estímulo a determinado grupo. As medidas do $\mathrm{VEF}_{1}$ foram feitas em equipamento com registro gráfico e análise computadorizada dos critérios de aceitação e reprodutibilidade. Estes foram preenchidos em todos os casos. Sendo o VEF uma medida objetiva e reprodutível, concluímos por ausência de viés de avaliação em nosso estudo.

A randomização neste estudo foi bem sucedida, não havendo diferenças significativas nos sinais clínicos de gravidade e no grau de obstrução ao fluxo aéreo entre os dois grupos.

Q uando os grupos de estudo não são semelhantes no início, apesar da randomização, em alguma característica que possa afetar os resultados, deve-se retrospectivamente tentar controlar o viés de confusão na análise dos resultados. Os grupos foram comparáveis em várias características, porém em algumas foram observadas diferenças. Isso seria esperado pelo grande número de comparações feitas. Pelo questionário aplicado, maior número de pacientes no grupo $\mathrm{F}$ referia duração menor do que 2 horas do efeito de broncodilatador utilizado nas últimas 6 horas antes da vinda à emergência (duração $=2$ horas, 37/ $46(80 \%)$ no grupo F vs. 22/ 41 $(54 \%)$ no grupo BIF, $\chi^{2}=7,12 p=0,05$ ) e maior percentagem de expectoração não mucóide - 22/ 40 (55\%) pacientes no grupo $\mathrm{F}$ e $10 / 36$ (28\%) no grupo $\mathrm{BIF}, \chi^{2}=5,76, \mathrm{p}=$ 0,05 . Esses dados indicam possível maior grau de inflamação no grupo F, com menor taxa de resposta neste grupo e, portanto, favorecimento de resposta para o grupo combinado. U ma análise de covariância, entretanto, não demonstrou influência desses fatores na magnitude da resposta funcional.

Diversos estudos avaliaram o efeito da adição do BI a $\beta_{2^{-}}$ agonistas na crise de asma. Em muitos destes, foi avaliado 0 
efeito adicional de dose única de ipratrópio ao $\beta_{2}$-agonista. 0 esquema terapêutico atualmente universal de doses repetidas de $\beta_{2}$-agonista em curto prazo não foi considerado em vários trabalhos. O desfecho avaliado quase sempre envolveu a resposta funcional, após um ou mais intervalos de tempo, seguindo-se a administração das drogas. A magnitude da eventual maior resposta funcional no grupo tratado com a combinação de broncodilatadores deveria resultar em impacto clínico, isto é, menor número de tratamentos adicionais necessários e menor percentagem de admissões hospitalares.

U ma revisão sistemática recente de quatro estudos envolvendo crianças e adolescentes tratados com múltiplas doses de broncodilatadores ${ }^{(26)}$ concluiu que a adição do BI pode evitar a admissão hospitalar em 1 de cada 11 pacientes tratados, porém o intervalo de confiança de $95 \%$ foi largo (de 5 a 250 crianças tratadas para evitar uma internação), refletindo o pequeno número de casos estudados. Em crises leves $\left(\mathrm{VEF}_{1}>55 \%\right)$ não haveria benefício.

U m grande estudo recente(27) envolvendo 434 crianças concluiu que na presença de crise grave (PFE $\leq 50 \%$ ou alto escore de gravidade), a adição de ipratrópio reduz significativamente a necessidade de hospitalização (de 53 para 38\%). U ma internação seria evitada para cada 7 tratamentos feitos com a combinação.

Karpel et al. ${ }^{(28)}$ randomizaram 384 pacientes adultos com $\mathrm{VEF}_{1} \leq 60 \%$ para dois tratamentos (192 em cada grupo), com albuterol $2,5 \mathrm{mg}$ isolado ou misturado com $0,5 \mathrm{mg}$ de ipratrópio, por nebulização, fornecidos inicialmente e após 45 minutos. Esse estudo foi englobado nos resultados relatados por Lanes et al.(17). A pós 90 minutos o $\mathrm{VEF}_{1}$ elevou-se $0,68 \mathrm{~L}$ no grupo que recebeu tratamento combinado e 0,65 no grupo tratado apenas com albuterol (NS). Antes da alta, 54\% (104) dos pacientes no grupo albuterol/ ipratrópio e $60 \%$ (116) dos pacientes no grupo albuterol $(p=0,22)$ necessitaram mais tratamentos na emergência. No grupo ipratrópio/ albuterol, 24 pacientes $(12,5 \%)$ foram internados; no grupo do albuterol, 26 pacientes (13,5\%) foram admitidos (NS). 0 objetivo principal desse estudo foi avaliar a resposta funcional e critérios para a internação não foram estabelecidos a priori, sendo os pacientes, após as duas doses de broncodilatadores, tratados de acordo com "a rotina da emergência".

Lin et al. ${ }^{(29)}$ rando mizaram 55 adultos com asma, com PFE $<200 \mathrm{~L} / \mathrm{min}$, para tratamento com albuterol $(2,5 \mathrm{mg}$ a cada 20 minutos, por três doses, $\mathrm{n}=28$ ) ou com albuterol no mesmo esquema associado a ipratrópio $(0,5 \mathrm{mg}$ combinado apenas com a dose inicial de albuterol, $N=27$ ). 0 objetivo primário do estudo foi avaliar mudanças do PFE com os tratamentos. A proporção de admissões nos dois grupos foi examinada como desfecho secundário. A despeito de um valor inicial menor para o pico de fluxo, os valores após os tratamentos foram maiores no grupo ipratrópio/ albuterol (mediana: 60 vs. $48 \%$ ). No grupo tratado apenas com albuterol
$10(36 \%)$ pacientes foram admitidos comparados com $3(11 \%)$ do grupo ipratrópio/ albuterol $(p=0,03)$. Diversos critérios de internação foram "formulados por consenso" na emergência, porém estes não incluíram valores para o PFE; os critérios aplicados em cada caso não foram relatados.

Em adultos, apenas um estudo realizado foi publicado em 1997 , envolvendo doses repetidas de $\beta_{2}$-agonistas e avaliando como desfecho a necessidade de internações ou de tratamento adicional em pacientes tratados na emergência. McFadden et al.(30) realizaram um estudo não randomizado, seqüencial, com pacientes tratados nos primeiros três meses com albuterol, 2,5mg por nebulização, a cada 20 minutos por três doses e nos três meses seguintes com a adição de $0,5 \mathrm{mg}$ de ipratrópio adicionado à primeira dose do albuterol. A pós a terceira dose, os pacientes poderiam ter alta na ausência de sinais de gravidade e PFE $\geq 60 \%$ do previsto. Os dois grupos não diferiram em suas características iniciais, exceto pela idade maior no grupo do ipratrópio. 0 estudo envolveu 131 doentes tratados com a combinação, comparados com 123 no grupo do albuterol. Pacientes com PFE > $50 \%$ foram incluídos. A pós o terceiro tratamento inalado, na ausência de resposta, aminofilina e corticóide IV eram fornecidos. 0 tempo de permanência na emergência não diferiu entre os dois grupos; igualmente, a percentagem de doentes admitidos foi semelhante $(28 \%$ no grupo do ipratrópio e $25 \%$ no grupo sem ipratrópio). 0 menor número de pacientes internados nesse estudo em relação ao nosso pode ser explicado pelo menor grau de obstrução na avaliação inicial.

0 presente estudo é o único que avaliou o efeito da adição do ipratrópio a $\beta_{2}$-agonista, utilizando nebulímetro pressurizado, acoplado a espaçador, combinação de comprovada eficácia na crise de asma ${ }^{(7)}$. A resposta funcional observada no presente estudo é de magnitude semelhante à da verificada em outros estudos utilizando nebulizadores; estes têm débito altamente variável e resposta pobre ou ausente em casos individuais pode resultar de sua utilização.

A aplicação de um protocolo sistemático de avaliação e tratamento da crise de asma na emergência resulta em redução importante das internações, melhor desfecho para as crises e grande economia de recursos ${ }^{(31)}$. No Brasil, o custo do ipratrópio é aproximadamente 10 vezes maior do que o do fenoterol.

Concluímos, com base nos dados obtidos, que a adição de ipratrópio a doses repetidas de $\beta_{2}$-agonista não tem efeito terapêutico adicional de impacto significativo em adultos, tratados na emergência, com crise grave.

\section{REFERÊNCIAS}

1. British Thoracic Society. The British guidelines on asthma management. 1995 review and position statement. Thorax 1997;52:S1-19.

2. National Institutes of Health; National Heart, Lung and Blood Institute. Guidelines for the Diagnosis and Management of Asthma. NHLBI/ WHO Workshop Report. Publication 1997;97:4051A. 
3. Beveridge RC, Grunfeld AF, Hodder RV, et al. Guidelines for the emergency management of asthma in adults. Can Med Assoc J 1996;155: 25-27.

4. National Institutes of Health; National Heart, Lung and Blood Institute. Global Initiative for Asthma. Global Strategy for Asthma Management and Prevention, NHLBI/WHO Workshop Report Publication 1995;95:3659.

5. Lipworth BJ, Newnham DM, Clark RA, et al. Comparison of relative airways and systemic potencies of inhaled fenoterol and salbutamol in asthmatic patients. Thorax 1995;50:54-61.

6. Tang OT, Flatley MA. Comparison of effects of inhaling a combined preparation of fenoterol with ipratropium bromide (Duovent) with those of fenoterol and salbutamol. Postgrad Med J 1984;60(Suppl 1):2427.

7. Turner MO, Patel A, Ginsburg S, et al. Bronchodilator delivery in acute airflow obstruction. A meta-analysis. Arch Intern Med 1997; 157:1736-1744.

8. Freitag A, Newhouse MT. Management of asthma in the 1990s. In: Tierny DF. Current pulmonology. Saint Louis: Mosby, 1994;19-74.

9. Colacone A, Afilalo M, Wolkove $\mathrm{N}$, et al. A comparison of albuterol administered by metered dose inhaler (and holding chamber) or wet nebulizer in acute asthma. Chest 1993;104:835-841.

10. Idris $\mathrm{AH}$, Mcdermott MF, Raucci J $\mathrm{C}$, et al. Emergency department treatment of severe asthma: metered-dose inhaler plus holding chamber is equivalent in effectiveness to nebulizer. Chest 1993;103:665672.

11. Elwood RK, A bboud RT. The short-term bronchodilator effects of fenoterol and ipratropium in asthma. J Allergy Clin Immunol 1982;69: 467-473.

12. Gomm AS, Keaney NP, H unt LP, et al. Dose-response comparison of ipratropium bromide from a metered-dose inhaler and by jet nebulization. Thorax 1983;38:297-301.

13. Allen CJ, Campbell AH. Dose response of ipratropium bromide assessed by two methods. Thorax 1980;35:137-139.

14. Gross NJ, Co E, Skorodin MS. Cholinergic bronchomotor tone in COPD: estimates of its amount in comparison to normal. Chest 1989; 96:984-987.

15. Rebuck AS, Chapman KR, Abboud R, et al. Nebulized anticholinergic and sympathomimetic treatment of asthma and chronic obstructive airways disease in the emergency room. Am J Med 1987;82:59-64.

16. Kelly HW, Murphy S. Should anticholinergics be used in acute severe asthma? Ann Pharmacother 1990;24:409-416.

17. Lanes SF, Garret JE, Wentworth CE, et al. The effect of adding ipratropium bromide to salbutamol in the treatment of acute asthma. Chest 1998;114:365-372.
18. American Thoracic Society - Standardization of spirometry - 1987 update. Am Rev Resp Dis 1987;136:1285-1296.

19. Pereira CAC, Barreto SP, Simões J G, et al. Valores de referência para a espirometria em uma amostra da população brasileira adulta. J Pneumol 1992;18:10-22.

20. Gardner RM, Crapo RO, J ackson BR, et al. Evaluation of accuracy and reproducibility of peak flow meters at 1,400 m. Chest 1992;101: 948-952.

21. Dompeling $E$, Van Schayck CP, Molema J, et al. A comparison of six different ways of expressing the bronchodilating response in asthma and COPD; reproducibility and dependence of prebronchodilator FEV ${ }_{1}$. Eur Respir J 1992;5:975-981.

22. Weir DC, Burge PS. Measures of reversibility in response to bronchodilators in chronic airflow obstruction: relation to airway calibre. Thorax 1991;46:43-45.

23. Higgins RM, Stradling J R. Nebulized anticholinergic and sympathomimetic treatment of asthma and chronic obstructive airways disease in the emergency room. Am J Med 1987;83:809-810.

24. British Thoracic Society and Associations of Respiratory Technicians and Physiologists. Guidelines for the measurement of respiratory function. Respir Med 1994;88:165-194.

25. Corbridge TC, Hall JB. The assessment and management of adults with status asthmaticus. Am J Respir Crit Care Med 1995;151:12961316.

26. Plotnick LH, Ducharme FM. Should inhaled anticholinergics be added to $\beta 2$ agonists for treating acute childhood and adolescent asthma? A systematic review. BMJ 1998;317:971-977.

27. Qureshi F, Pestian J, Davis P, Zaritsky A. Effect of nebulized ipratropium on the hospitalization rates of children with asthma. $\mathrm{N}$ Engl J Med 1998;339:1030-1035.

28. Karpel JP, Schacter EM, Fanta C, et al. A comparison of ipratropium and albuterol vs albuterol alone for the treatment of acute asthma. Chest 1996;110: 611-616.

29. Lin RY, Pesola GR, Bakalchuk L, et al. Superiority of ipratropium plus albuterol over albuterol alone in the emergency department management of adult asthma: a randomized clinical trial. Ann Emerg Med 1998;31:208-213.

30. McFadden ER, EISanadi N, Strauss $L$, et al. The influence of parasympatholytics on the resolution of acute attacks of asthma. Am J Med 1997:102:7-13.

31. McFadden ER, Elsanadi N, Dixon L, et al. Protocol therapy for acute asthma: therapeutic benefits and cost savings. Am J Med 1995;99: 651-661. 